\title{
Band anti-crossing and carrier recombination in dilute nitride phosphide based lasers and light emitting diodes
}

\author{
James Chamings', Sucheta Ahmed', Alfred R. Adams', Stephen J. Sweeney, ${ }^{*}, 2$, \\ Vladimir A. Odnoblyudov ${ }^{3,4}$, Charles W. Tu ${ }^{4}$, Bernardette Kunert ${ }^{5,6}$, and Wolfgang Stolz ${ }^{6}$ \\ ${ }^{1}$ Advanced Technology Institute, University of Surrey, Guildford, Surrey, GU2 7XH, United Kingdom \\ ${ }^{2}$ Center for Nanophotonics and Electrical Engineering Department, Arizona State University, Tempe, Arizona 85287-8806, USA \\ ${ }^{3}$ Quanlight, 2223 Avenida de la Playa, Suite 212, La Jolla, California 92037, USA \\ ${ }^{4}$ Department of Electrical and Computer Engineering, University of California at San Diego, La Jolla, California 92093-0407, USA \\ ${ }^{5}$ NAsP III/V GmbH, 35041 Marburg, Germany \\ ${ }^{6}$ Material Sciences Center and Department of Physics, Philipps-University, Hans-Meerwein-Straße, 35032 Marburg, Germany
}

Received 18 June 2008, revised 6 November 2008, accepted 6 November 2008

Published online 24 December 2008

PACS 42.55.Px, 71.20.Nr, 71.55.Eq, 72.20.Jv, 85.60.Jb

* Corresponding author: e-mail s.sweeney@surrey.ac.uk, Phone: +44 1483 689406, Fax: +44 1483689404

We use high pressure techniques to investigate the properties of two classes of "dilute-nitride-phosphide"-based devices; $\mathrm{GaNP} / \mathrm{GaP}$ light emitting diodes for yellow-amber-red display applications and $\mathrm{GaNAsP} / \mathrm{GaP}$ lasers, a potential route to producing lasers monolithically on silicon. Based upon high pressure electroluminescence measurements we find that the band anti-crossing (BAC) model reasonably describes the $\mathrm{GaN}(\mathrm{As}) \mathrm{P}$ system based on an average of the nitrogen states. In terms of device characteristics, we find that carrier leakage into the X-minima of GaP reduces the efficiency of GaNP/ $\mathrm{GaP}$ LEDs with increasing pressure. Lasing has been observed in GaNAsP/GaP devices with a pulsed threshold current density of $2.5 \mathrm{kA} / \mathrm{cm}^{2}$ at $80 \mathrm{~K}(\lambda=890 \mathrm{~nm})$. A weak increase in threshold current with hydrostatic pressure indicates that a carrier leakage path that does not involve the GaP $X$-minima is the dominant carrier recombination mechanism in these devices, in contrast to the LEDs.
1 Introduction Over the last 15 years, research into so called "dilute nitride" material systems has generated much interest, due to the interesting physics and application for optoelectronic devices [1]. This includes a large band gap bowing and an increase in electron effective mass [2] with only a few percent of incorporated nitrogen, these in turn enable telecommunications wavelength lasers to be grown on GaAs substrates and the potential for more efficient solar cells [3]. More recently, attention has turned to GaP-based dilute nitrides where additional interesting phenomena and applications can also be explored. In this paper using high pressure techniques we investigate the validity of a simple band anti-crossing (BAC) model in describing GaP-based dilute nitride materials and consider the factors limiting the electro-optic efficiency of GaN(As)P LEDs and lasers based on GaP.

1.1 GaNP/GaP LEDs For over four decades nitrogen impurities have been added to the indirect semiconductor $\mathrm{GaP}$ to act as a radiative defect centre to produce light emitting diodes (LEDs) [4]. Such devices exploited the fact that Nitrogen forms localized levels within the forbidden band gap to increase the radiative efficiency of the indirect band gap host GaP [5]. In GaAs based dilute nitrides, the interaction between the localized $\mathrm{N}$ states and the conduction band (CB) minimum has been effectively modelled with both the BAC model and the empirical pseudopotential method [6]. This has recently led to renewed activity in 
dilute nitrides based on GaP. Yellow-amber-red GaNPbased LEDs are predicted to offer better device characteristics than current AlInGaP devices due to the weak temperature dependence of the band gap [7], their lower thermal resistivity [8] and the lower manufacturing costs associated with monolithic growth on GaP, providing a better and cheaper alternative to red LEDs in tri-colour white solid state lighting.

1.2 GaNAsP/GaP lasers The dominance of silicon (Si) for electronic and microelectronic circuit applications has lead to the search for monolithic optoelectronic integrated circuits (OEICs) on Si substrates. One of the key components of OEICs is a laser material for efficient light emission. However, the indirect band gap of $\mathrm{Si}$ has meant efficient light emission and gain have been difficult to achieve. Several strategies for producing lasers on silicon have been proposed [9], such as the "hybrid" laser [10], whereby an InP-based active region is wafer fused onto a silicon/silica based waveguide or utilizing the Raman Effect with external optical pumping [11]. However monolithic growth on a silicon substrate coupled with electrical injection has remained challenging. Due to their direct band gap, III-V based semiconductors such as GaAs and InP are the most commonly used materials to produce semiconductor lasers. Growth of conventional direct gap III-V compound semiconductors directly onto $\mathrm{Si}$ is very difficult due to the formation of threading dislocations as a result of the large lattice mismatch [12]. However, it has been shown that GaP can be grown on $\mathrm{Si}$ with a low dislocation density due to their relatively small difference in lattice constant $(<0.4 \%$ at room temperature) [13]. A GaNAsP alloy with high As fractions and dilute $\mathrm{N}$ fractions (of $\sim 4 \%$ ) can form a direct narrow band gap material approximately lattice matched to $\mathrm{GaP}$ and $\mathrm{Si}$ [14]. Hence the realization of a GaP-based direct band gap semiconductor laser material on a silicon substrate can provide a realistic route towards monolithic laser sources for silicon-based photonic/electronic circuits.

\section{Devices studied}

2.1 GaNP/GaP LEDs The GaNP/GaP LED devices studied here were grown by MBE and utilize simplified chip processing by one-step growth on transparent $350 \mu \mathrm{m}$ thick n-type $\mathrm{GaP}(100)$ substrates. The epitaxial layers consist of a $0.3 \mu \mathrm{m}$ thick $\mathrm{n}-\mathrm{GaP}$ layer ( $\mathrm{Si}$ doped), $0.15 / 0.1 / 0.15 \mu \mathrm{m}$ thick undoped $\mathrm{GaP} / \mathrm{GaN}_{0.006} \mathrm{P}_{0.994} / \mathrm{GaP}$ active region, and $0.8 \mu \mathrm{m}$ thick $\mathrm{p}-\mathrm{GaP}$ (Be doped) contact layer. LED chips were fabricated using $\mathrm{Ge} / \mathrm{Au} / \mathrm{Ni} / \mathrm{Au}$ and $\mathrm{AuZn}$ metallization for $\mathrm{n}$ - and $\mathrm{p}$-type contacts respectively.

2.2 GaNAsP/GaP lasers The GaNAsP/GaP laser samples studied consist of a single $6 \mathrm{~nm} \mathrm{GaN}{ }_{0.04} \mathrm{As}_{0.8} \mathrm{P}_{0.16} /$ GaP 2.5\% compressively strained $\mathrm{QW}$ grown by MOVPE. Optical confinement is provided by $\mathrm{Al}_{0.23} \mathrm{Ga}_{0.77} \mathrm{P}$ cladding layers. The devices were fabricated using a typical broad contact edge-emitting laser process. The device stripes (50 $\mu \mathrm{m}$ and $100 \mu \mathrm{m}$ wide) were defined using regular masks and standard contacts were formed by metal deposition. The devices were measured as-cleaved with cavity lengths of $980 \mu \mathrm{m}$ and had an emission wavelength of $890 \mathrm{~nm}$ at $80 \mathrm{~K}$.

3 Experimental techniques The devices were driven under pulsed operation with $100 \mathrm{~ns}$ long pulses at a duty cycle of $10 \mathrm{kHz}$ in order to minimize Joule heating effects. Electroluminescence (EL) from the devices was collected using a broad area Si detector. For the lasers, the threshold current was measured from the facet light versus current characteristic. We also investigated the temperature and current dependencies of the pure spontaneous emission spectra from which we extracted the radiative component of the threshold current. To measure the spontaneous emission, we milled a window in the n-doped side of the devices using a focused ion beam technique and aligned an optical fibre. This technique is described in detail elsewhere [15]. An optical spectrum analyzer was used to collect the spontaneous emission from both the LEDs and lasers.

Hydrostatic pressure measurements were also performed on the devices using a 3 stage compression system coupled with a $\mathrm{CuBe}$ pressure cell. This allowed pressures of $0-10 \mathrm{kbar}$ using gaseous helium as the pressure medium. The pressure was measured by a manganin coil in the final compression piston. Electrical access was provided using Vespel seals and a sapphire window allowed optical access. Low temperature pressure measurements $(\sim 100 \mathrm{~K})$ were realised by placing the pressure cell in a liquid nitrogen bath. The device temperature was confirmed by comparing the atmospheric pressure peak EL wavelength against the known temperature dependence of the emission peak.

4 Application of the Band Anti-Crossing (BAC) model This simple model [16] describes the effect of nitrogen incorporation in dilute nitride alloys and allows the prediction of temperature and pressure dependencies of $\mathrm{CB}$ states. In the BAC, the interaction between a single localized $\mathrm{N}$ state and extended $\mathrm{CB}$ states splits the $\mathrm{CB}$ into two sub-bands, $E_{+}$and $E_{-}$. Their energies may be described by the following equation:

$$
E_{\mathrm{g} \pm}(P)=\frac{1}{2}\left[\left(E_{\Gamma}(P)+E_{\mathrm{N}}\right) \pm \sqrt{\left(E_{\Gamma}(P)-E_{\mathrm{N}}\right)^{2}+4 x C_{\mathrm{NM}}^{2}}\right] .
$$

Here $E_{\Gamma}(P)$ is the host material pressure dependent $\Gamma$ conduction band edge, $E_{\mathrm{N}}$ is the energy level of the localized $\mathrm{N}$ state, $x$ is the $\mathrm{N}$ fraction and $C_{\mathrm{NM}}$ is the coupling parameter determined by the strength of coupling between localized and extended states. All of these energies are defined with respect to the zone center valence band (VB) maximum. $E$ is therefore taken as the "band gap" energy and compared to either the peak EL for the LEDs or the lasing energy extracted from pressure dependent EL measurements. The $E_{+}$ level is not observed in these measurements but has been shown in other studies [17]. 
4.1 GaNP/GaP LEDs Shown in Fig. 1 is the pressure dependence of the EL spectra (top) and peak energy position (squares), taken from the EL spectra of the GaNP LEDs (bottom). Also shown are the pressure dependencies of the $\Gamma$ - and $X$-minima of GaP. The emission peak blue shifts with increasing pressure at a rate of $+1.6 \mathrm{meV} / \mathrm{kbar}$, consistent with previous spectroscopic [5] and theoretical [6] studies. It can be clearly seen that the change of the peak energy does not correlate with either minima, suggesting that the emission peak is strongly influenced by the addition of nitrogen. It is therefore interesting to see if the BAC model can describe this pressure dependence. The nitrogen level, $E_{\mathrm{N}}$, was found from photocurrent $(\mathrm{PC})$ measurements (as in Peternai et al [18]) and determined to be $2.21 \mathrm{eV}$, consistent with other findings [5, 17] and shows negligible change with pressure, as previously observed [19] and calculated theoretically [6] for isolated nitrogen impurities in GaP. It is clearly seen in Fig. 1 that the BAC model gives good agreement with the experimental data
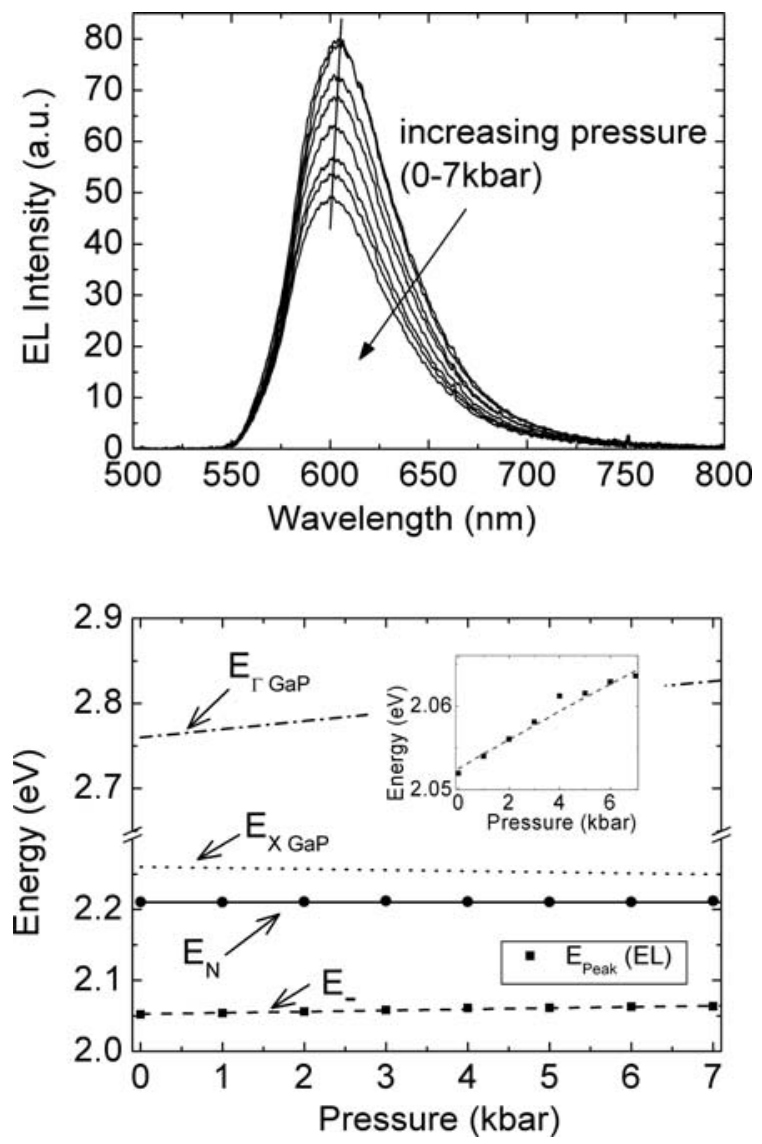

Figure 1 Measured EL spectra for pressures 0-7 kbar (top) and the pressure dependence of peak EL (squares) for GaNP LEDs (bottom). Also shown is the pressure dependence of $\Gamma_{\mathrm{GaP}}$ (dotdashed line), $X_{\text {GaP }}$ (dotted line). The nitrogen level position (solid line) was determined by PC measurements (circles) and show negligible movement with pressure. The change in peak EL with pressure can be predicted using the BAC model (dashed). The insert shows the fit in greater detail. for which $C_{\mathrm{NM}}=4.38 \mathrm{eV}$. This is larger than values for the GaInNAs/GaAs system for which $C_{\mathrm{NM}}$ is typically between $1.2 \mathrm{eV}$ and $2.7 \mathrm{eV}[20,21]$, since $E_{\mathrm{N}}$ and $\Gamma_{\text {GaP }}$ are further apart and $E_{\mathrm{N}}$ is below the lowest conduction band states in GaP. We note here that $C_{\mathrm{NM}}$ is usually determined by growing a set of samples with varying alloy fraction and $C_{\mathrm{NM}}$ may itself vary with alloying. By applying pressure, we effectively apply a small perturbation to the host band gap and hence can use it as an accurate way of probing the $\mathrm{N}-$ level - host conduction band coupling and determine a reliable estimate of $C_{\mathrm{NM}}$.

4.2 GaNAsP/GaP lasers Shown in Fig. 2 is the pressure dependence of the EL spectra (top) and lasing energy (squares), taken from EL spectra of a GaNAsP lasers (bottom), which increases with pressure at a rate of $5.0 \mathrm{meV} / \mathrm{kbar}$. Also shown is the pressure dependence of the $\Gamma$ - and $X$-minima of the host material $\mathrm{GaAs}_{0.84} \mathrm{P}_{0.16}$, determined from a linear interpolation of $\mathrm{GaAs}$ and $\mathrm{GaP}$ [22].
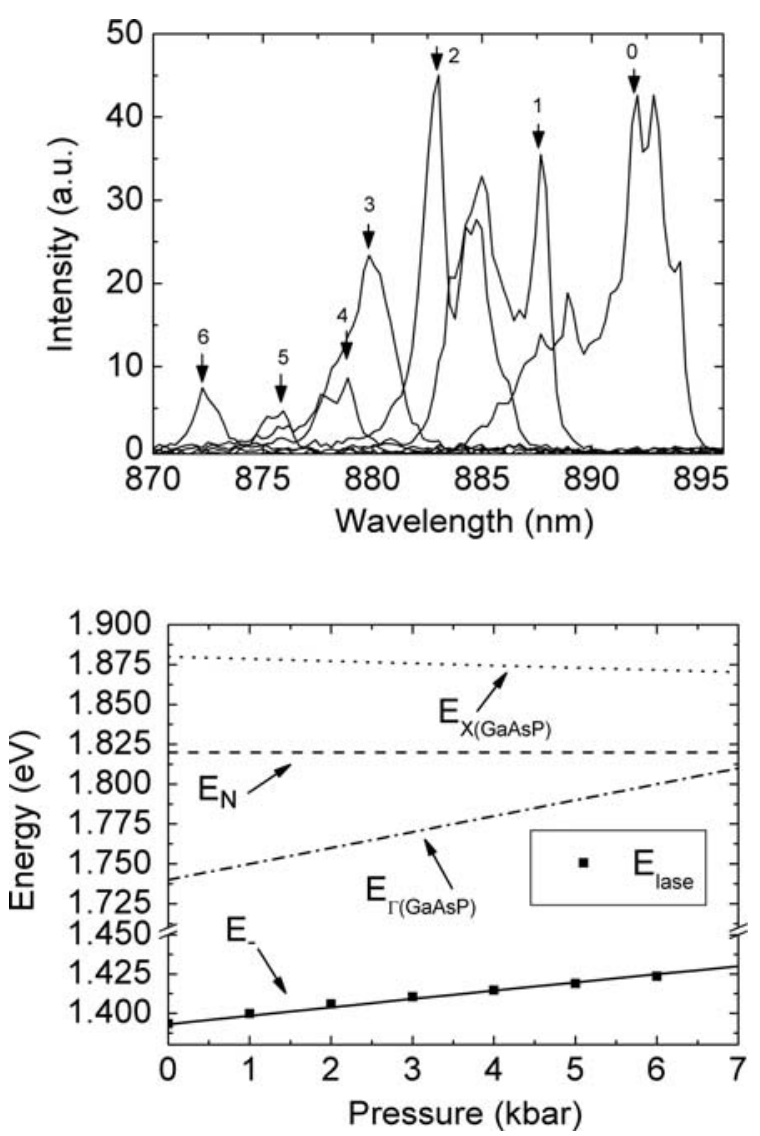

Figure 2 Measured EL spectra for pressures 0-6 kbar (top) and the pressure dependence of the lasing energy (squares) for GaNAsP SQW lasers (bottom). Also shown is the pressure dependence of $\Gamma_{\text {GaAsP }}$ (dot-dashed line) and $X_{\text {GaAsP }}$ (dotted line). The nitrogen level (dashed line) position was determined by interpolation of isolated $\mathrm{N}$ level in GaAs and $\mathrm{GaP}$ and assumed negligible movement with pressure. The change in lasing energy with pressure can be predicted using the BAC model (solid line). 
$E_{\mathrm{N}}$ was found from interpolation of the isolated nitrogen level in $\mathrm{GaAs}$ and $\mathrm{GaP}$ and has been assumed to have a negligible pressure dependence (as observed in Section 4.1).

The BAC model gives good agreement with the experimental data for which $C_{\mathrm{NM}}=1.9 \mathrm{eV}$. This is much lower than that for the GaNP LEDs and may be attributed to the fact that the separation between the Nitrogen level and the conduction band edge of the host matrix is much smaller for the GaNAsP devices as compared to the GaNP LEDs. Based upon these results we conclude that while it is well known that nitrogen induces multiple localized levels in III-V semiconductors, a simple two-level BAC model based on an "average" of the nitrogen levels provides a useful predictive tool for the pressure dependence of the peak emission for dilute-nitride-phosphide based devices at room temperature and, hence, may be useful for tuning the properties of dilute nitride devices based upon $\mathrm{GaP}$ materials.

\section{Pressure dependent device characteristics}

5.1 GaNP/GaP LEDs In order to optimise the efficiency of GaNP based LEDs it is important to understand the processes which limit device efficiency. The close proximity of the $X$-minima in particular may give rise to carrier leakage in GaP-based LEDs. The importance of the GaP $X$-minima can be determined with the use of high pressure. We may assume that carriers can flow via two paths; radiatively producing a photon, or thermally leaking into the indirect barrier regions and recombining nonradiatively. Since pressure reduces the $\Gamma-X$ inter-valley separation, it can be used to probe the importance of this leakage path. In the presence of a leakage process which increases with pressure, the radiative component of the injected current, $I_{\text {rad }}$ associated with the light emission may be written as

$$
I_{\mathrm{rad}}=I_{0} \exp \left(\frac{\mathrm{d} \Delta E}{\mathrm{~d} P} \frac{P}{k T}\right)
$$

where $I_{0}$ is a constant, $\mathrm{d} \Delta E / \mathrm{d} P$ is rate of change of the conduction band quasi Fermi-level to leakage level separation with pressure, $k$ is the Boltzmann constant, $T$ is the absolute temperature and $P$ is the pressure. Here, the pressure dependence of the conduction band quasi Fermi-level is assumed equal to the emission peak shift. The pressure coefficient of the $X$-minima in GaP is also well known $\left(\mathrm{d} E_{X(\mathrm{GaP})} / \mathrm{d} P=-1.5 \mathrm{meV} / \mathrm{kbar}\right) \quad[23]$. Figure 3 shows the measured pressure dependence of the integrated EL spectra (normalized at atmospheric pressure) for a GaNP device at a fixed current of $10 \mathrm{~mA}$. The decrease in EL intensity with pressure is strong evidence that carrier leakage into the $X$-minima is significant. From earlier, we found the pressure coefficient of the emission peak energy to be $+1.6 \mathrm{meV} / \mathrm{kbar}$, also given that the pressure coefficient of the $X$-minima in $\mathrm{GaP}$ is known to be $-1.5 \mathrm{meV} / \mathrm{kbar}$, we deduce that, $\mathrm{d} \Delta E / \mathrm{d} P=-3.1 \mathrm{meV} / \mathrm{kbar}$. Substituting this value of $\mathrm{d} \Delta E / \mathrm{d} P$ into Eq. (2) yields the solid line shown in

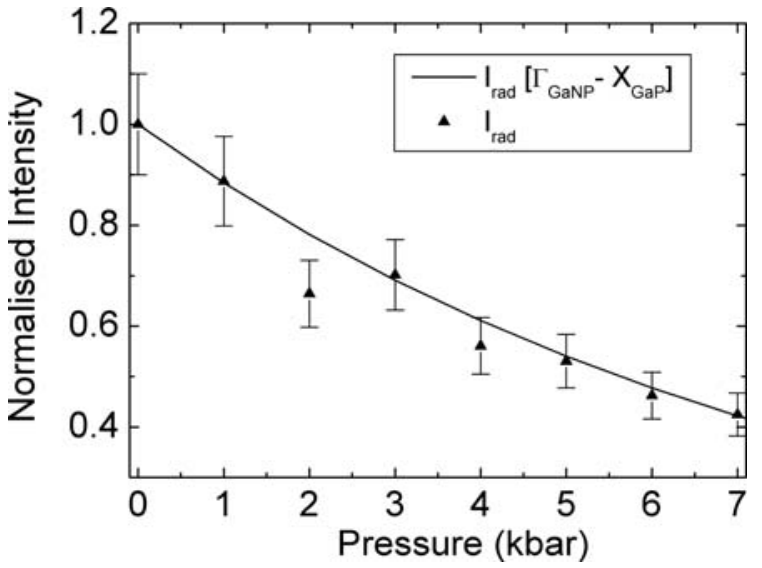

Figure 3 Normalized intensity versus pressure at a fixed current for GaNP LEDs. The solid line shows the calculated change in normalized intensity due to electron leakage into $X_{\mathrm{GaP}}$.

Fig. 3. This provides excellent agreement with the experimental data confirming that leakage into the $X$-minima of $\mathrm{GaP}$ is the important non-radiative recombination process in these devices.

5.2 GaNAsP/GaP lasers We find that these ascleaved devices have threshold current densities of $2.5 \pm 0.5 \mathrm{kA} / \mathrm{cm}^{2}$ with a lasing wavelength of $890 \mathrm{~nm}$ at $80 \mathrm{~K}$. This value of $J_{\text {th }}$ is much larger than well established GaAs-based lasers, which operate at similar wavelengths for which $J_{\mathrm{th}} / Q W \sim 100-200 \mathrm{~A} / \mathrm{cm}^{2}([24,25]$ and Refs. therein), the cause of which became the focus of our investigations. Figure 4 shows the measured pressure dependence of $J_{\text {th }}$ for the GaNAsP devices at $100 \mathrm{~K}$. Also shown is the ideal expected variation [26] of $J_{\text {rad }} \propto E_{\mathrm{g}}^{2}$ according to simple theory, where $E_{\mathrm{g}}$ is taken to be the lasing energy (from $E_{\text {lase }}=h c / \lambda_{\text {lase}}$, where $\lambda_{\text {lase }}$ is the measured lasing wavelength). Note that in each case, $J_{\text {th }}$ and $J_{\text {rad }}$ have been normalized to their respective values at atmospheric pressure. Clearly, it can be seen that $J_{\text {th }}$ increases more rapidly with pressure than the ideal $J_{\text {rad }}$, showing an increase in non-radiative recombination with increasing pressure. This may occur if carriers escape into a leakage level, $E_{\text {leak }}$, which, in relative terms, moves closer to the conduction band edge with increasing pressure. Assuming diffusive leakage, the corresponding leakage current [27] may be written as

$$
J_{\text {leak }}=J_{0} \exp \left(-\frac{\mathrm{d} \Delta E}{\mathrm{~d} P} \frac{P}{k T}\right),
$$

where $J_{0}$ is a constant, $\Delta E$ is the energy separation between the conduction band quasi Fermi-level and the leakage level, $k$ is Boltzmann's constant, $T$ is the absolute temperature and $P$ is the pressure. Thus, by considering the change in $\Delta E$ with pressure, $\mathrm{d} \Delta E / \mathrm{d} P$, the responsible leakage level may be identified from its pressure coefficient. From Fig. 4, a good fit is obtained for $\mathrm{d} \Delta E / \mathrm{d} P=-0.7 \mathrm{meV} / \mathrm{kbar}$. Since, from Fig. 2, the measured increase in band gap with pres- 


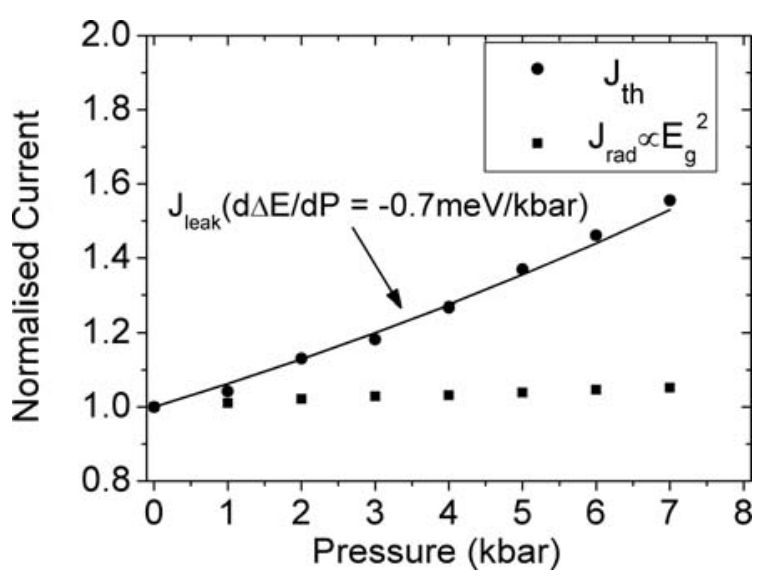

Figure 4 Measured pressure dependence of $J_{\text {th }}$ (circles) and $J_{\text {rad }}$ (squares) $\propto E_{\mathrm{g}}^{2}$ dependence for the GaNAsP devices, at $100 \mathrm{~K}$, normalized at atmospheric pressure. The solid line shows calculated change in threshold current with $\mathrm{d} \Delta E / \mathrm{d} P=-0.7 \mathrm{meV} / \mathrm{kbar}$.

sure is $+5.0 \mathrm{meV} / \mathrm{kbar}$, and assuming that the conduction band quasi Fermi-level has a similar pressure dependence, this corresponds to a pressure coefficient of $+4.3 \mathrm{meV} /$ kbar for the leakage level. Hence we conclude that the leakage level has a similar pressure dependence to the conduction band quasi-Fermi level. This suggests that unlike the GaNP LEDs, leakage into the $X$-minima of the indirect barriers is not significant in these devices, as one might expect due to the larger separation between the $E_{-}$ level and the $X$-minima in the longer wavelength GaNAsP lasers. Further work is ongoing to determine the exact leakage route within the laser heterostructure. If these leakage paths can be reduced, the growth of a direct bandgap material on GaP and eventually Si substrates may lead to fully monolithic silicon-based optoelectronic integrated circuits operating at room temperature.

6 Conclusions In summary, we have presented high pressure investigations into the material properties and device characteristics of GaNP-based LEDs and GaNAsPbased lasers. In spite of the multiplicity of nitrogen levels we find that the BAC model can reasonably be used as a predictive tool to describe these dilute-phosphide-nitride materials. Furthermore, high pressure measurements have shown that whilst carrier leakage into the $X$-minima of the GaP limits the efficiency of the LEDs, the lasers operating at longer wavelengths are dominated by a different leakage path, the exact mechanism of which is currently the subject of further investigation. The data shows there is still scope for optimisation of these materials which offer great potential for the development of more efficient semiconductors for display and silicon-compatible laser applications.

Acknowledgements The authors gratefully acknowledge EPSRC and the $\mathrm{SET}^{2}$ consortium for financial support, and the Deutsche Forschungsgemeinschaft (DFG) within the Topical Research Group 483 "Metastable Compound Semiconductor Systems and Heterostructures". The work at UCSD was supported by the von Liebig Center for Entrepreneurism and Technology Advancement, UCSD.

\section{References}

[1] M. Kondow, K. Uomi, A. Niwa, T. Kitatani, S. Watahiki, and Y. Yazawa, Jpn. J. Appl. Phys. 35, 1273 (1996).

[2] I. Vurgaftman and J. R. Meyer, J. Appl. Phys. 94, 3675 (2003).

[3] S. R. Kurtz, A. A. Allerman, E. D. Jones, J. M. Gee, J. J. Banas, and B. E. Hammons, Appl. Phys. Lett. 74, 729 (1999).

[4] R. Z. Bachrach, W. B. Joyce, and R. W. Dixon, J. Appl. Phys. 44, 5458 (1973).

[5] W. Shan, W. Walukiewicz, K. M. Yu, J, Wu, J. W. Ager III, H. P. Xin, and C. W. Tu, Appl. Phys. Lett. 76, 3251 (2000).

[6] P. R. C. Kent and A. Zunger, Phys. Rev. B 64, 115208 (2001).

[7] V. A. Odnoblyudov and C. W. Tu, J. Vac. Sci. Technol. B 24, 2202 (2006).

[8] S. Adachi, J. Appl. Phys. 54, 1844 (1983).

[9] P. Bell, Nature 409, 974 (2001).

[10] M. N. Sysak, H. Park, A. W. Fang, J. E. Bowers, R. Jones, O. Cohen, O. Raday, and M. J. Paniccia, Opt. Express 15, 15041 (2007).

[11] H. Rong, R. Jones, A. Liu1, O. Cohen, D. Hak, A. Fang, and M. Paniccia, Nature 433, 725 (2005).

[12] S. F. Fang, K. Adomi, S. Iyer, H. Morkoc, H. Zabel, C. Choi, and N. Otsuka, J. Appl. Phys. 68, 31 (1990).

[13] H. Yonezu, Semicond. Sci. Technol. 17, 762 (2002).

[14] B. Kunert, K. Volz, J. Koch, and W. Stolz, Appl. Phys. Lett. 88, 182108 (2006).

[15] S. J. Sweeney, A. F. Philips, A. R. Adams, E. P. O'Reilly, and P. J. A. Thijs, IEEE Photonics Technol. Lett. 10, 1076 (1998).

[16] C. Skierbiszewski, Semicond. Sci. Technol. 17, 803 (2002).

[17] I. A. Buyanova, M. Izadifard, A. Kasic, H. Arwin, W. M. Chen, H. P. Xin, Y. G. Hong, and C. W. Tu, Phys. Rev. B 70, 85209 (2004).

[18] L. Peternai, J. Kovac, J. Jakabovic, A. Vincze, A. Satka, and V. Gottschalch, Vacuum 80, 229 (2005).

[19] B. Gil, M. Baj, J. Camassel, H. Mathieu, C. Benoit à la Guillaume, N. Mestres, and J. Pascual, Phys. Rev. B 29, 3398 (1984).

[20] W. Shan, W. Walukiewicz, J. W. Ager III, E. E. Haller, J. F. Geisz, D. J. Friedman, J. M. Olson, and S. R. Kurtz, Phys. Rev. Lett. 82, 1221 (1999).

[21] P. Perlin, P. Wisniewski, C. Skierbiszewski, T. Suski, E. Kaminska, S. G. Subramanya, E. R. Weber, D. E. Mars, and W. Walukiewicz, Appl. Phys. Lett. 76, 1279 (2000).

[22] S. Wei and A. Zunger, Phys. Rev. B 60, 5404 (1999).

[23] C. Jauberthie-Carillon and C. Guillemin, J. Phys.: Condens. Matter 1, 6807 (1989).

[24] H. Li, T. Truchan, D. Brown, R. Pryor, R. Pandey, F. Reinhardt, J. Mott, G. Treusch, and S. Macomber, Opt. Laser Technol. 36, 327 (2004).

[25] G. Adolfsson, S. M. Wang, M. Sadeghi, and A. Larsson, Electron. Lett. 43, 454 (2007).

[26] S. J. Sweeney, D. McConville, N. F. Massé, R.-X. Bouyssou, A. R. Adams, C. N. Ahmad, and C. Hanke, Phys. Status Solidi B 241, 3391 (2004).

[27] D. Lock, S. J. Sweeney, A. R. Adams, and D. J. Robbins, Phys. Status Solidi B 235, 542 (2003). 